\title{
PROKLA-Redaktion
}

\section{Vom Gelde}

Unter dern unscheinbaren Titel »Vom Gelde« erschien im Jahre 1931 (ein Jahr nach dem englischen Original) das erste $»$ große $\ll$ Werk von Keynes, in dem er die orthodoxe Auffassung des Geldes kritisierte. Davon ausgehend legte er dann 1936 seine »General Theory« vor, die zu dem vielleicht einflußreichsten wirtschaftstheoretischen Werk dieses Jahrhunderts wurde, was aber nicht verhinderte, daß sie vom gegenwärtigen neoklassischen Mainstream als im wesentlichen überholt betrachtet wird.

Nicht viel anders erging es einem anderen Autor, der gut 70 Jahre vor Keynes auch zunächst eine Schrift veröffentlichte, die die vorherrschenden Geldtheorien (und zwar sowohl der »bürgerlichen « Ökonomen als auch der sozialistischen Autoren seiner Zeit) kritisierte und einige Jahre später mit einer breit angelegten Analyse und Kritik kapitalistischer Produktionsverhältnisse an die Öffentlichkeit trat: die Rede ist natürlich von Karl Marx und seiner 1859 erschienen Schrift $\gg$ Zur Kritik der politischen Ökonomie « und dem 1867 folgenden ersten Band des »Kapital «.
Daß Marx und Keynes - die großen Häretiker der ökonomischen Theorie - die beiden einzigen relevanten Alternativen zur klassisch-neoklassischen Verharmlosung der ökonomischen Bedeutung des Geldes entwickelten, wurde auch schon im Editorial der PROKLA 63 festgehalten, die vor 10 Jahren mit dem schlichten Titel »Geld《 erschien. Inzwischen ist aber durch die tatsächliche ökonomische Entwicklung vielleicht noch deutlicher geworden, daß gilt: »money matters «. Im Prozeß der deutschen Vereinigung spielte die Währungsunion sicher eine wichtigere Rolle als die politische Vereinigung. Was die EU angeht, so ist auch hier die geplante Währungsunion zu dem Thema schlechthin geworden, das alles andere in den Schatten stellt. Die Krisen des Europäischen Währungsssystems 1992 und 1992, die zum Ausstieg von England und Italien führten, gehörten ebenso wie die Krise des mexikanischen Pesos 1994/95 zu den wichtigsten weltwirtschaftlichen Ereignissen der letzten Jahre. Wäre Geld tatsächlich nur »numéraire « und bloBes »Schmiermittel « für die 
»realen « Transaktionen - wie es die neoklassische Orthodoxie will - so ließe sich die ganze Aufregung kaum verstehen.

Aber in welcher Weise kommt es dazu, daß »money matters $\ll$ ? In welcher Beziehung stehen »reale und »monetäre « Sphäre? Mit dieser Frage setzen sich die beiden Artikel von Robert Guttmann und von Michael Heine und Hansjörg Herr auseinander. Während Guttmann der These von der Entkopplung realer und monetärer Akkumulation aufgrund einer Analyse des Zerfalls fordistischer Regulation des Finanzsystems zuneigt, wird diese Auffassung von Heine und $\mathrm{Herr}$ vehement kritisiert. Dabei reicht ihre Kritik bis zur Marxschen Theorie, der sie aufgrund ihrer paradigmatischen Struktur die Möglichkeit einer adäquaten Darstellung der modernen monetärer Phänomene rundweg absprechen. Hier sollte die Diskussion sicherlich noch fortgeführt werden.

Mit den sich gerade neu abzeichnenden Formen des »cybermoney «, des elektronischen Geldes, dem in
Zukunft wahrscheinlich eine erhebliche Bedeutung zukommen wird, beschäftigen sich die Aufsätze von Raúl Rojas und Elmar Altvater. Dabei stehen in dem Artikel von Rojas die technischen Aspekte dieser neuen Form des Geldes im Vordergrund, während Altvater das Computergeld im Rahmen globaler Finanzinnovationen betrachtet und damit auch einen Beitrag zur Debatte über das Verhältnis von monetärer und realer Akkumulation leistet.

Thomas Achim Werner untersucht in seinem Aufsatz die ökonomischen Auswirkungen der Geldwäsche, die längst kein begrenztes Phänomen mehr ist, sondern Konsequenzen für die gesamte Ökonomie eines Landes haben kann.

Außerhalb des Schwerpunkts erscheint die schon länger angekündigte Fortsetzung des Artikels von Wolf-Dieter Narr und Roland Roth aus Heft 100, die damit die Diskussion um ein den gegenwärtigen Problemen angemessenes Sozialismusverständnis fortführen. 